\title{
PROPOSICIONES COMPLETIVAS Y ESTRUCTURAS ALTERNANTES. SISTEMA Y NORMA EN EL ESPAÑOL DE CORRIENTES (ARGENTINA)
}

\author{
Ofelia Kovacci \\ Universidad de Buenos Aires \\ Consejo de Investigaciones Cientificas y Técnicas
}

\section{INTRODUCCIón}

Trabajamos sobre un corpus de habla de la provincia de Corrientes con conversaciones acerca de temas de interés antropológico o sociológico. Dentro de él aparecen espontáneamente las construcciones que nos ocupan. Se trata del sistema sintáctico-semántico formado por varias estructuras vinculables por relaciones de paráfrasis:

a) las proposiciones sustantivas encabezadas con el incluyente que, tradicionalmente llamadas "completivas". Estas proposiciones se integran como sujeto, objeto, etc., en predicaciones que manifiestan la llamada "modalidad explícita" 1 . La predicación conforma el modus y la proposición es el dictum;

b) las construcciones parentéticas, en las que varias clases de predicados incluyentes de a) se intercalan en el constituyente dictal o lo siguen, sin conexión sintáctica ${ }^{2}$;

1 Charles Bally, «Syntaxe de la modalité explicite», Cabiers Ferdinand de Saussure, 2, 1942, págs. 3-4.

2 J. O. Urmson, *Parenthetical verbs», Mind, 61, 1952, págs. 480-496. 
c) los modificadores de la modalidad ${ }^{3}$, en los que varias clases de predicados incluyentes de a) forman una construcción periférica ${ }^{4}$;

d) el llamado estilo directo, introducido por clases específicas de predicados de a).

Concluimos con el examen de las normas en la construcción de las proposiciones completivas.

Nuestro corpus ${ }^{5}$ se basa en dieciocho horas de grabación en la ciudad de Corrientes (C) y aledaños (Cr) y en los departamentos de San Luis del Palmar (SLP), Bella Vista (BV), Concepción (Co), Mercedes (M), Saladas (S), Paso de los Libres (PL), Esquina (E), Curuzú Cuatiá (CC), Goya (G), Monte Caseros (MC), San Miguel (SM), Santo Tomé (ST) e Ituzaingó (I). En las grabaciones intervienen 64 hablantes, de los cuales 13 son maestros y coordinadores de alfabetización (hablantes de nivel B), y el resto alfabetizandos (hablantes A). Del total de informantes mencionados, 45 produjeron en forma espontánea las construcciones que estudiamos ${ }^{6}$.

\section{Sistema}

\subsection{Proposiciones completivas}

Estas proposiciones aparecen como sujeto, objeto directo o predicativo. Las predicaciones ${ }^{7}$ que se registran son las siguientes ${ }^{8}$.

3 O. Kovacci, «Modificadores de modalidad», en su Estudios de gramática española, Buenos Aires, Hachette, 1986, págs. 89-95.

- O. Kovacci, «La jerarquía de las funciones sintácticas», Boletín de la Academia Am gentina de Letras, LIV, n. 213-214, 1989, págs. 377-384.

s Es parte del material recogido en 1986 y 1988 en centros de alfabetización por Nilda D. Solís y Lila Petrella, para el proyecto del Atlas Lingǘstico-Antropológico de la República Argentina. Cf. Documentos del PREDAL, Argentina, 2, Buenos Aires, Ministerio de Educación y Justicia-OEA, 1987.

- Este número se reparte del siguiente modo según las variables de nivel, edad y sexo:

\begin{tabular}{|c|c|c|c|c|}
\hline \multirow{3}{*}{$\frac{\operatorname{Edad} \ldots \ldots \ldots}{\text { Sexo } \ldots \ldots \ldots}$} & \multicolumn{3}{|c|}{ A } & \multirow{2}{*}{$\frac{B}{25-45}$} \\
\hline & $15-25$ & $26-50$ & más de 50 & \\
\hline & $\mathrm{H} \quad \mathrm{M}$ & $\mathrm{H} \quad \mathrm{M}$ & H M & $\mathrm{H} \quad \mathrm{M}$ \\
\hline & 34 & $\begin{array}{ll}7 & 13\end{array}$ & 35 & 55 \\
\hline
\end{tabular}

7 Cf. Tracy D. Terrell, «Assertion and presupposition in Spanish complements», en Current studies in Romance languages, ed. por M. Luján y F. Hensey, Washington D. C., Georgetown University Press, 1976, págs. 221-245; O. Kovacci, «Proposiciones relativas discontinuas, extraposición del relativo y la distribución de los modos en la inclusión sustantivaw, en su Estudios de gramática española, cit. en nota 3.

- En los ejemplos se identifica la localidad, el nivel y el sexo. La variable edad no se 
De reporte o expositivas:

(1) Me mandó a decir que había una familia $[\ldots]$ que lo quería a el (MC, Am).

(2) Los mismo alumno manifestaron que ello aprendian ... (BV, Bm).

(3) Yo le enseño que ella tiene que obedecer (C, Bf).

(4) Conversó que $[\ldots]$ llegó un tiempo que ya nadie la podía curar (C, Af).

(5) Yo le cuento a ello que le ha dado una flor (M, Af).

De opinión:

(6) Yo creo que de todo hay que saber (C, Af).

(7) Yo pienso que muy abandonada está la gente de campo (C, Af).

(8) Calculo que [el río] se lleva para arriba [el cuerpo] (C, Am).

(9) $\mathrm{Si}$ me atiende otro doctor se me hace que no me va a curar (I, Af).

(10) Parece ser que yo desde ese momento me quedé sin vista (C, Af).

(11) Resulta que él tenía una novia (G, Am).

(12) Habia sido que sabla; por eso me curó (E, Af).

(13) Imaginese que nadie quiere ir (BV, Af).

(14) Está demostrado por la historia que han errado (CC, Bm).

(15) La verdad que hoy la gente está muy dispierta ( $G, A m)$.

(16) Claro que la persona que mandó hacer una red tiene que arreglarla también $(\mathrm{C}, \mathrm{Am})$.

De conocimiento y percepción:

(17) Usted tiene miedo porque sabe que ahf le va a salir un perro (G, Am).

(18) Yo be visto que uno de mis primos tenía una reliquia $(\mathrm{G}, \mathrm{Am})$.

(19) Yo siento que ... yo quiero volver a ese lugar (G, Af).

Factivas emotivas:

(20) Sería muy bueno que acá tuviéramos una cooperativa (SM, Af).

(21) Es raro eno es cierto? que los de la isla no tengan que saber hablar perfectamente el guarani; es raro (I, Af).

(22) Suerte que tenía esta habilidad de trabajar ( $\mathrm{Cr}, \mathrm{Af})$.

Volitivas :

(23) No queremo que el hijo entienda en guaranf (C, Af).

(24) Esperamos que la casa sea nuestra (G, Af).

corresponde con diferencias apreciables en el corpus. Marcamos con $b$ la aspiración de $s$ implosiva ante vocal. 
(25) Le pedimos que ore (G, Bf).

(26) Dejamo que la hoja largue el jugo (C, Af).

(27) El me dijo que le dé el jarabe (C, Af).

De duda :

(28) Nivel de quince, veinte ano, puede que se vuelque alll $(\mathrm{C}, \mathrm{Bm})$.

El modo del verbo de la proposición se ajusta a la rección estándar ${ }^{9}$, excepto en un caso en que aparece el indicativo en lugar del subjuntivo:

(29) Dicen que tiene, pero yo no be visto que tiene (G, Am).

Hay muestras en B de los grados que puede manifestar el modo verbal en la proposición cuando la matriz es de opinión:

(30) Como correntina yo pienso que se debiera cultivar más el idioma guaraní (ST, Af).

El subjuntivo debiera ocupa en una escala de 'certeza' el polo - , frente a debe que se acerca al polo + .

En cuanto al tiempo de la proposición, también la correlación es estándar. Se observa que la perspectiva temporal de futuro de los verbos incluidos en matrices volitivas de pretérito tiende a una correlación abierta, en subjuntivo, cuando la acción (proceso, estado) puede continuarse en el futuro del emisor :

(31) El me dijo que le dé el jarabe (C, Af).

Dé aparece en lugar de diera.

Es frecuente la construcción impersonal de la estructura incluyente con verbos expositivos en tercera persona plural (cf. texto $29 \|$ : Dicen que ...).

\subsection{Construcción parentética}

Una forma de indicar la fuente y la responsabilidad de una aseveración es presentar la predicación modal como adjunción parentética, eliminando el conector de inclusión. Entre la estructura del tipo considerado en 1.1 y la parentética hay una relación sintáctico-semántica constante.

- O. Kovacci, op. cit. en nota 7. 
En el corpus la expresión parentética se construye con predicaciones de opinión, reporte y desconocimiento:

(32) Le mataron -creo-. Le mataron ahi [al gaucho Gil] (G, Af).

(33) Una cosa $1 \ldots$ que se da mucho es que ... diriamos, acá hay una naturalidad. Usted va a Mercedes y es lo mismo (CC, Bm).

(34) A pesar de que los remedios, dicen -yo no sé- que están hechos de los yuyos ... (C, Af).

En este ejemplo se combina la construcción completiva (dicen ... que) y la expresión parentética (yo no sé), que salvaguarda la responsabilidad del hablante frente a la aseveración que propone la construcción impersonal (dicen ...). En 35 se acumulan dos adjuntos; el primero, de opinión, es inmediatamente rectificado por el de desconocimiento:

(35) Uno está trabajando [...]; el gobierno lo tiene que ayudar en algo, pienso yo, no sé (G, Am).

\subsection{Modificadores de modalidad}

Son las expresiones que indican el alcance que el hablante otorga a su actitud ante el enunciado y, por consiguiente, a los términos del enunciado mismo ${ }^{10}$.

En los textos siguientes la aseveración se apoya en la percepción (ver) o el conocimiento (saber) no necesariamente completo del hablante, quien por ello apela retóricamente a la conformidad del interlocutor:

(36) Como veo, es, es ... asi ... una pavadita ... (C, Af).

(37) Hasta donde yo sé, ¿no es cierto?, una mujer no más se lastimó (I, Am).

(38) Que yo sepa, víctimas fatales, como se dice, no hubo (I, Am).

En el último ejemplo hay un segundo modificador de modalidad (como se dice) cuyo dominio es fatales, según el cual se corrobora la elección del vocablo, en conformidad con el decir común (de hablantes no especificados, dada la forma pasiva impersonal se dice). El mismo tipo de modificador modal de conformidad cita el decir del propio hablante como apoyo cierto de la aseveración:

(39) Como le estábamo diciendo, no" ayudamo no mán (E, Am).

(40) Asi como le digo, pescamo unos pescaditon $(\mathrm{E}, \mathrm{Am})$.

10 Esta función se evidencia en las paráfrasis que relacionan la construcción con las de 1.1 (sin implicar, naturalmente, correspondencia semántica total):

Como veo, es, es... asf ... una pavadita ... / Veo que es, es... 
El modificador de conformidad - también construido en este caso como impersonal- establece con su encabezador según mayor distancia que como respecto de la aseveración del emisor actual y atenúa su compromiso con el contenido del enunciado:

(41) Según dicen, le gusta [al lobisón] comer criatura recién nacida (I, Am).

\subsection{Estilo directo}

En el corpus analizado la mención icónica ${ }^{11}$ de oraciones o discursos se introduce con verbos de comunicación lingüística y de operación mental, sin conector. Típicamente en las hablas A aparece el verbo decir, del primer tipo. Su colocación es variable:

(42) Un día me dice el padre: «Me voy a verle a un hombre, a ver qué tiene» [el niño] (E, Af).

(43) «Tienen segundo grao, pero segundo grao de antes», dicen ello" (SLP, Am).

(44) - La nena - me dijo el hombre - no le vaya a poner en la cama porque se va a caer (C, Af).

En B también es frecuente el estilo directo:

(45) Le repito: hemos publicado en Clarin (C, Bm).

(46) Algunas maestras decian: «No, porque la alfabetización es sólo de grandes.» Otros pensábamos: «No, porque ... esa participación pudo ser de una familias (G, Bf).

Con el mismo valor de pensar, denotativo de operación mental, del ejemplo precedente, también se emplea decir en A :

(47) Y ... digo yo: «Si yo me voy por acá, ahl hay más monte» (E, Af).

11 Nos referimos a una representación construida por el hablante, no necesariamente idéntica al texto original, conservando rasgos propios del campo mostrativo de ese texto y su entonación. Los verbos que introducen la proposición se limitan a los de reporte y los volitivos que contienen los rasgos 'comunicación, lingǘfstica'. En algunos casos el rasgo 'comunicación' se cambia por 'operación mental' (pensar, reflexionar, etc.). 


\subsection{Frecuencia de las construcciones}

El conjunto de las construcciones del sistema examinado ${ }^{12}$ que se registran en el corpus, suma 199 ocurrencias. Estas se reparten del siguiente modo: a) completivas, $129(64 \%) ; b)$ estilo directo, $43(22 \%) ; c)$ parentéticas, 15 $(8 \%) ; d)$ modificadores de modalidad, $12(6 \%)$.

$\mathrm{Si}$ bien no tenemos datos de las frecuencias en otros dialectos, creemos que las indicadas para el habla correntina reflejan una proporción normal de ocurrencia de los miembros del sistema.

\section{NORMAS}

\subsection{Construcción de las proposiciones completivas}

Las construcciones que hemos enumerado se ajustan al sistema de la lengua general. En los registros de nuestro corpus transcriptos en 1.1, las proposiciones sustantivas (declarativas) se conectan con el incluyente que, forma normal recomendada por la Academia ${ }^{13}$ y textos escolares. B. Vidal de Batti$\mathrm{ni}^{14}$ observa el "uso de un $d e$ superfluo que se considera incorrecto", frecuente "en el habla popular de Buenos Aires y su gran zona de influencia". Este uso, llamado dequeismo, "suele oírse hasta en personas cultas y es un hábito muy arraigado particularmente en la capital y en todo el litoral". Kany ya había notado el fenómeno en Hispanoamérica y citaba antecedentes peninsulares ${ }^{15}$.

Mencionan usos dequeístas en la Argentina Donni de Mirande ${ }^{16}$ para Ro-

13 Construcciones que no pertenecen al sistema, pero que presentan proposiciones sus. tantivas con que, son ciertas oraciones ecuacionales y formas de relieve:

Hay dos cosas: una es que el puestero... va siendo cada vez menos necesario (CC, Am).

Por eso que los días de Navidad y Afio Nuevo se trabaja con el auto (I, Am).

Cf. O. Kovacci, «Sobre la estructura de la forma de relieve con ser y proposición relativa», Voz y Letra, II/1, 1991, págs. 45-46.

13 Real Academia Española, Esbozo de una nueva gramática de la lengua española, Ma. drid, $1973 ; 3.19 .9$ n. 1 .

14 Berta E. Vidal de Battini, El español de la Argentina, Buenos Aires, Consejo Nacional de Educación, 1964, pág. 195.

${ }_{16}$ Charles E. Kany, American Spanish syntax, Chicago \& London, The University of Chicago Press, Second Edition, 1951, pág. 353.

${ }_{16}$ Nélida E. Donni de Mirande, El español hablado en Rosario, Rosario, Facultad de Filosoffa, UNL, 1968, pág. 173. 
sario, Rojas ${ }^{17}$ para Tucumán, E. Garcia ${ }^{18}$ para la ciudad de Buenos Aires, Fontanella de Weinberg ${ }^{19}$ para el sur bonaerense (ciudad de Bahía Blanca). Las publicaciones más recientes con estudios sistemáticos son las de Boretti de Macchia ${ }^{20}$, referentes al habla culta de Rosario. En el corpus oral de la autora ${ }^{21}$ las formas dequeistas alcanzan el $5 \%$ y los hombres "superan ampliamente a las mujeres" en este caso, "particularmente los de primera y segunda generación".

En nuestro corpus de la provincia de Corrientes contamos 17 registros de dequeísmo (todos en alternancia con la forma canónica), de los cuales 16 fueron emitidos por informantes varones de veintiocho a cuarenta años. Sólo un registro pertenece al habla $\mathrm{A}$ (lo mismo que la única emisión femenina). Se emplean en la construcción las siguientes predicaciones:

(48) Cree que le aparece algo y no es nada... La gente creen de que es mal agüero $(\mathrm{G}, \mathrm{Am})$.

(49) Es aconsejable de que no se deje de leer ( $\mathrm{Co}, \mathrm{Bm})$.

(50) Se va viendo de que eso va mejorando (CC, Bm).

(51) Sabia de que si hay una organización mínima en el barrio le damo a la gente del barrio que se vayan apoyando $(i b$.).

(52) Uno va descubriendo de que la misma persona va teniendo una pila de oficios $(i b$.).

(53) Me dijeron que no era casada (G, Af).

(54) Me dicen de que tengo que ser casada (ib.).

Existen varias teorías para explicar el fenómeno del dequeísmo. Vidal de Battini no ofrece ninguna explicación; pero Kany atribuye el dequeísmo a una antigua confusión del régimen de preposiciones, llevada a América, que abarca tanto la supresión como la adición de preposiciones. Da ejemplos de uso dequeísta ocasional en Sánchez de Badajoz y en Pereda, y agrega que este empleo "se basa posiblemente en el ritmo sintáctico". Donni de Mirande y Rojas mencionan la antigüedad del uso.

17 Elena M. Rojas, Aspectos del babla en San Miguel de Tucumán, Tucumán, Universidad Nacional de Tucumán, 1980, págs. 202-203.

18 Erica C. García, «El fenómeno (de)queismo desde una perspectiva dinámica del uso comunicativo de la lengua», Actas del II Congreso Internacional sobre el Español de América, ed. por José G. Moreno de Alba, México DF, UNAM, 1986, págs. 56-57.

19 María Beatriz Fontanella de Weinberg, El español bonaerense, Buenos Aires, Hachette, 1987.

${ }_{20}$ Susana H. Boretti de Macchia, «(De)queísmo en el habla culta de Rosario», Anuario de Lingǘstica Hispánica, V, 1989, págs. 27-48; «(Des)uso preposicional en el habla culta: queismo y dequefsmo*, en Nélida E. Donni de Mirande et al., Variación lingǘftica en el español de Rosario, Universidad Nacional de Rosario, Consejo de Investigaciones, 1991, págs. 115-123.

n En *(Des)uso preposicional en el habla culta: quelsmo y dequef́smo», cit. en n. 18; pág. 118. 
Dado que el fenómeno tiene una extensión considerable en América, ha merecido estudios detallados, como los de Rabanales ${ }^{22}$, Bentivoglio ${ }^{23}$ y Arjona ${ }^{24}$, para Chile, Venezuela y Méjico, respectivamente. Rabanales trata la "inestabilidad normativa" (que / de que) como consecuencia de cruces de regimenes diferentes (temer que / temor de que).

Bentivoglio y D'Introno 25 sugieren que "la inserción de de [ante que] modifica la interpretación semántica de la oración", pues "debilita la aserción" cuando los verbos incluyentes son asertivos (reporte, opinión) o "atenúa la presuposición" en los casos de matrices factivas. En un trabajo posterior Bentivoglio ${ }^{26}$ abandona esta interpretación, y sobre la base de variables sociolingüísticas trata el dequeísmo como un fenómeno de ultracorrección. Formula la hipótesis de que

la corrección sistemática del queismo [en la escuela] lleva a ciertos hablantes a asociar la presencia de la preposición ante que SUB con formas de habla más educada y refinada. Los hablantes de nivel medio, cuya inseguridad lingüistica es bien conocida, tienden a la ultracorrección y utilizan la forma que ellos creen más prestigiosa —en este caso de que $\mathrm{SUB}-$.

Esto ocurre porque los hablantes asocian el prestigio lingüístico a los medios de ascenso social. En Caracas los hombres son más dequeístas que las mujeres, y según Bentivoglio, el fenómeno del dequeísmo se estaba propagando hacia el nivel alto (más seguro lingüísticamente por su inserción en un nivel elevado de la escala social), sin alcanzar aún el nivel bajo.

De modo semejante a la situación caraqueña descripta, en el corpus correntino la tendencia al dequeísmo se centra en los varones del nivel B. Habrá que estudiar la procedencia y la dirección de este proceso, que en zonas de irradiación no muestra diferencia tajante entre el nivel medio y el bajo ${ }^{27}$.

22 Ambrosio Rabanales, «Queísmo y dequelsmo en el español de Chile», en Estudios filológicos y lingüisticos. Homenaje a Angel Rosenblat en sus 70 años, Caracas, Instituto Pedagógico, 1974, págs. 413-444.

2s Paola Bentivoglio, «Queísmo y dequefsmo en el habla culta de Caracas», en Frances M. Aid et al. (comp.), 1975 Colloquium on Hispanic Linguistics, Washington D. C., Georgetown University Press, 1976, págs. 1-18.

24 Marina Arjona, *Anomallas en el uso de la preposición de en el español de México», Anuario de Letras, XVI, 1978, págs. 67-90; «Usos anómalos de la preposición de en el habla popular mexicana», Anuario de Letras, XVII, 1979, págs. 167-184.

${ }_{23}$ Paola Bentivoglio y Francesco D'Introno, «Análisis sociolingüístico del dequeísmo en el habla de Caracas», Boletín de la Academia Puertorriqueña de la Lengua Española, VI, 1977, págs. 58-82.

28 Paola Bentivoglio, «El dequeísmo en Venezuela: ¿un caso de ultracorrección?», en Homenaje a Ambrosio Rabanales, BFUCh, XXXI, 1980-1981, págs. 712-718.

27 Así lo sugieren ejemplos cotidianos, como son los siguientes, ofdos por TV en la ciudad de Buenos Aires (mayo de 1992):

Quiero significarle de que se vende más diario (Diariero).

Pienso de que hay libertad de prensa (Periodista politico). 
E. García ${ }^{28}$ adoptó la hipótesis de Bentivoglio-D'Introno acerca de la diferenciación semántica que acarrearian las formas con preposiciones respecto de las que la omiten. El contenido seria en el primer caso "el distanciamiento del hablante, que no quiere comprometerse totalmente con el contenido de la cláusula" ${ }^{2}$. En contrario puede aducirse el discurso de un hablante que describe rasgos de la cultura correntina y los defiende por ser valiosos, y al mismo tiempo emite buena cantidad de formas dequeistas ${ }^{30}$ que, en varios casos - según la teoría de García - le harían "tomar distancia" de aquello que precisamente defiende. Así lo muestran los siguientes textos $(\mathrm{CC}, \mathrm{Bm})$ :

(55) Yo le digo de que en el aspecto social, económico, hay un esfuerzo.

(56) A nosotros nos gusta comentarlo de que en toda la comunidad [la cultura] tiene matrices muy de esa época.

(57) Cuando el Municipio no le puede dar, entonces le enseñamo de que vaya a Cáritas.

(58) Yo creo de que en eso estamos de vuelta.

(59) Siempre piensa de que la gente ... no borre, sino al contrario apoye todo este basamento que nosotros tenemos.

(60) Asf que eso demostró de que esa es la solución.

(61) Nosotros vemos de que tiene vigencia [la cultura guaranf].

(62) ... tenerlo en cuenta, sf, de que ... hay mucho ${ }^{h}$ oficio ${ }^{h} \ldots$ por ejemplo el tema del bordado.

(63) Nosotro hacemos de que por ahi nos den algunas cosas, pero la mano de obra la ponemo nosotros.

Este hablante casi ha automatizado de que como locución, puesto que la aplica hasta a un que consecutivo:

(64) Los vascos mismos se acorrentinaron tanto, de que iban ... eh ... las palabras vascas se fueron este... guaranizando.

El proceso de ultracorrección tiende a volverse automático.

\subsection{Hipótesis sobre la forma canónica}

$\mathrm{El}$ alto porcentaje de formas canónicas en el corpus $(86,66 \%)$ y el escaso número de hablantes dequeístas (dos en $\mathrm{A}$; tres en $\mathrm{B}$ ) merecen evaluarse

Hemos descubierto de que conversando... (Arbitro de fútbol).

Creo de que nuestro reclamo son justo (Delegado obrero).

Creo de que hay que actualizar la Constitución (Abogado).

28 V. op. cit. en nota 18.

29 Boretti de Macchia se ha esforzado por justificar esta posición en 1989; pero adopta una actitud más cauta en 1991 (trabajos citados en la nota 20).

so Sobre siete ocurrencias con el verbo creer, en cinco emplea de que. 
dentro de las características generales del español de Corrientes, que forma parte de una zona americana de antiguo contacto español-guaraní.

Por una parte, habrá que considerar paralelismos y contrastes, puesto que el habla correntina ha conservado en todos sus niveles ciertos rasgos tradicionales castellanos a diferencia de otras hablas de la Argentina: la consonante lateral palatal, el leísmo, una mayor vigencia del pretérito perfecto compuesto de indicativo.

Por otra parte, habrá que examinar la estructura del guaraní, que interfiere en diversos aspectos del habla de las zonas de contacto ${ }^{31}$. En esta lengua las proposiciones sustantivas presentan tres posibilidades de construcción ${ }^{32}$ :

a) la proposición se yuxtapone al predicado incluyente, sin conector:

$$
\begin{array}{ll}
\text { rei-mo?ấ } & \text { se- tavś } \\
\text { tú cre(er) } & \text { yo soy tonto } \\
\text { tú crees que yo soy tonto }
\end{array}
$$

b) el conector (pospuesto) es há o hagwé, a veces precedido de artículo (el préstamo la):

he-?í o-ù hagwé

él dice él ven(ir) que

dice que viene

c) el conector de 'propósito' es hayzwá, con matrices volitivas:

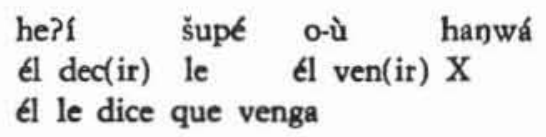

El conector de b) y c) no se acumula con ningún otro elemento subordinante. Este rasgo, así como el caso a), pueden haber ayudado a sostener el empleo de la forma canónica (que encabezador de proposiciones sustantivas).

\footnotetext{
s1 Véase Germán de Granda, «Convergencia lingüística en el bilingüismo paraguayo», Serta Pbilologica F. Lázaro Carreter, I, Madrid, Cátedra, 1983.

33 Emma Gregores y Jorge A. Suárez, Adescription of colloquial Guarani, The HagueParis, Mouton \& Co., 1967, págs. 157-158.
} 